\title{
Associations of Depression and Anxiety with Stigma in a Sample of Patients in Saudi Arabia Who Recovered from COVID-19
}

\author{
Munirah A Alkathiri $\mathbb{D}^{1,2}$, Omar A Almohammed $\mathbb{D}^{1,2}$, Faleh Alqahtani $\mathbb{D}^{3}$, Yazed AlRuthia $\mathbb{D}^{1,2}$ \\ 'Department of Clinical Pharmacy, College of Pharmacy, King Saud University, Riyadh, Saudi Arabia; ${ }^{2}$ Pharmacoeconomics Research Unit, Department \\ of Clinical Pharmacy, College of Pharmacy, King Saud University, Riyadh, Saudi Arabia; ${ }^{3}$ Department of Pharmacology and Toxicology, College of \\ Pharmacy, King Saud University, Riyadh, Saudi Arabia \\ Correspondence: Yazed AIRuthia, Tel +996 II4677483; +966 509726340, Fax +966 II4677480, Email yazeed@ksu.edu.sa
}

\begin{abstract}
Purpose: The coronavirus disease (COVID-19) outbreak combined with social distancing, isolation, and movement restrictions has had a profound impact on individuals' physical and psychological well-being. The aim of this study was to examine the associations of depression and anxiety with feelings of stigma among patients in Saudi Arabia who have recovered from COVID-19.

Materials and Methods: A cross-sectional survey was conducted between July and December 2020. Trained healthcare providers contacted and interviewed participants by phone. Depression, anxiety, and stigma were assessed using the Patient Health Questionnaire (PHQ-9), the Generalized Anxiety Disorder (GAD-7) questionnaire, and the Explanatory Model Interview Catalogue stigma scale (EMIC). Data on sociodemographic characteristics, comorbidities, and family history of mental illness were also collected. Multiple linear regression models were performed to explore factors associated with depression and anxiety.

Results: A total of 174 adult participants ( $\geq 18$ years old) who had recently recovered from COVID-19 were interviewed. The mean PHQ-9 and GAD-7 scores were $7.53( \pm 5.04)$ and $3.77( \pm 4.47)$, respectively. About $68 \%$ of the participants had at least mild depression (PHQ-9 score of 5-9), whereas only $29.89 \%$ had at least mild anxiety (GAD-7 score of 5-9) during their infections with COVID-19. Multiple linear regression showed that females were more vulnerable to depression and anxiety disorders than their male counterparts were $(\beta=3.071$ and $\beta=1.86$, respectively). Notably, participants' stigma scores were significantly associated with higher scores on depression and anxiety.

Conclusion: These findings highlight the negative consequences of COVID-19 infection on the mental health of recovered patients. Therefore, considerable attention from local and international health authorities is needed to improve the mental well-being of recovered COVID-19 patients.
\end{abstract}

Keywords: mental health, depression, anxiety, stigma, Saudi Arabia

\section{Introduction}

The coronavirus disease (COVID-19) pandemic has had unprecedented detrimental effects on healthcare systems worldwide, resulting in physical and mental-health consequences, ${ }^{1,2}$ which can be profound and long-lasting. ${ }^{3}$ Moreover, COVID-19 has provoked fear of an imminent economic crisis and recession. ${ }^{2}$ To prevent the spread of the virus, various preventive measures have been implemented, such as maintaining social distance, isolation, and movement restrictions. ${ }^{4}$ However, these measures combined with the continuous spread of the virus have profoundly changed people's lifestyles and negatively affected their mental health and well-being. ${ }^{5,6}$

Several psychological and behavioral changes have been observed during this and previous outbreaks of infectious diseases and these behavioral changes have had critical effects on the dynamics of the outbreaks by altering the diseases' characteristics, such as level of severity, transmission, and consequences at the individual, national, and international levels. ${ }^{7}$ For instance, during the Ebola outbreak, individuals were predicted to experience fear of suffering or having pain, dying, feeling hopeless, and being stigmatized by others. ${ }^{8}$ Likewise, the COVID-19 pandemic has led to marked 
behavioral changes, which can lead to psychological disorders. ${ }^{9}$ Health crises, such as the COVID-19 pandemic, have been associated with negative mental health outcomes among healthcare providers and the general population, which are often expressed as fear, anxiety, depression, and stigmatization. ${ }^{10}$ Identifying these psychological changes and disorders in the early stages of a disease may result in the implementation of interventions that are more effective in containing the psychological consequences of pandemics. ${ }^{10}$

Major depression is a prevalent and recurrent mood disorder associated with persistent feelings of sadness that leads to reduced productivity and quality of life and increased morbidity and mortality. ${ }^{11,12}$ It has been ranked by the World Health Organization as one of the main causes of disability worldwide and is projected to be the top leading cause of disability by $2030{ }^{13,14}$ Anxiety disorders refer to a group of psychiatric disorders that produce fear, worry, and a constant feeling of being overwhelmed. ${ }^{15}$ In developing countries, approximately $10-44 \%$ of people experience symptoms of depression and anxiety, but less than $35 \%$ of them receive medical care. ${ }^{16}$ The COVID-19 pandemic represents a significant threat to psychological well-being and could significantly increase the incidence rates of depression and anxiety. ${ }^{17} \mathrm{~A}$ Chinese study found that approximately $20 \%$ of participants suffered from depressive symptoms and $35.1 \%$ from anxiety symptoms during the COVID-19 outbreak. ${ }^{18}$ Similarly, an increased prevalence of depression and anxiety were reported among the general population in the United Kingdom during the COVID-19 pandemic. ${ }^{19}$ Of equal importance, the experience of being stigmatized due to COVID-19 infection could lead to feelings of disgrace. ${ }^{20}$ Previous studies have reported an association between mental disorders and stigmatizing attitudes. For instance, Ociskova et al found that patients with psychiatric disorders, including anxiety and depression, were at a higher risk for self-stigmatization and discrimination. ${ }^{21}$ Another study found that having depression was significantly correlated with an increased level of perceived stigma. ${ }^{22}$ The relationship between stigma and health has been investigated more than a hundred years ago. ${ }^{23}$ Erving Goffman came up with the theoretical framework back in the late 1950s which laid out the relationship between stigma defined as a negative perception of the community toward certain individuals or group of people based on their social, physical, behavioral, or mental traits and mental illness in psychiatric hospitals. ${ }^{24}$ This theory which then became known as benchmark social theory of the relationship between stigma and disease is now used to explain the relationship between different physical and mental diseases and stigma. ${ }^{20}$ Undoubtedly, these negative and stigmatizing attitudes toward mental illness and people with psychiatric disorders are barriers to seeking help from healthcare professionals. ${ }^{25}$

The psychological impact of COVID-19 on affected individuals is a concerning global health issue, and studies indicate that those individuals are at a high risk for adverse mental health outcomes. ${ }^{26,27} \mathrm{~A}$ meta-analysis of the epidemiologic studies that examined the prevalence of mental illness during the COVID-19 pandemic found that the infected patients, quarantined people, and patients with noncontagious chronic diseases were at greater risk for depression and anxiety compared to the rest of the population. ${ }^{28}$ Another study revealed that patients with COVID-19 infection had higher rates of symptoms of depression, anxiety, and post-traumatic stress disorder compared to their non-COVID-19 counterparts. ${ }^{29}$ Multiple factors were found to be related to the mental health issues of these vulnerable individuals, including self-isolation, hospitalization, stigma, and discrimination accompanied by the absence of social support. ${ }^{29-31}$ Moreover, individuals with a pre-existing or family history of mental illness were more likely to have adverse mental health outcomes during the COVID-19 pandemic. ${ }^{32,33}$

There is a paucity of research addressing the impact of COVID-19 on mental health in Saudi Arabia. Although feelings of stigma have been investigated among patients in Saudi Arabia who have recovered from COVID-19, including whether they felt stigmatized by the people around them, no studies have examined the associations of depression and anxiety with stigma in this segment of the population. ${ }^{33}$ Therefore, the aim of this study was to examine the association between depression/anxiety and stigma among patients with COVID-19 who had been infected with the disease and recovered from it during the COVID-19 pandemic in Saudi Arabia.

\section{Materials and Methods}

\section{Study Design and Setting}

A cross-sectional survey was conducted from July to December 2020. Adult ( $\geq 18$ years) Arabic-speaking patients who had recently recovered from symptomatic COVID-19 infection (eg, $\leq 14$ days) in Saudi Arabia were included in the 
study and asymptomatic patients were excluded. Participants were selected by a search query that retrieved random observations from the Ministry of Health $(\mathrm{MoH})$ database of patients with SARS-CoV-2 infection that was confirmed by a polymerase chain reaction (PCR) test. They were contacted and interviewed over the phone by one of four trained healthcare providers. Additionally, they were informed about the purpose of the study and their consent was obtained verbally since no written informed consent form was required.

\section{Ethical Approval}

The Kingdom of Saudi Arabia's MOH approved the study's protocol (IRB No: 20-11E/17-06-2020), and the ethics committee waived the requirement for patients' written informed consent to participate in the study since the waiver will not adversely affect the rights of the participants to withdraw from the study at any time during the interview, the data collection sheet did not include any personal identifiers, (eg, name, national ID, phone number, or address), and the study did not involve more than minimal risk to participants. The study was conducted in accordance with the ethical principles of the Helsinki declaration.

\section{Instruments and Measurements}

Three previously validated and translated assessment scales, namely, the Patient Health Questionnaire (PHQ-9), ${ }^{34,35}$ the Generalized Anxiety Disorder (GAD-7) questionnaire, ${ }^{35,36}$ and the Explanatory Model Interview Catalogue stigma scale $(\mathrm{EMIC})^{33,37}$ were used to assess depression, anxiety, and stigma, respectively. The Arabic version of the PHQ-9 scale is a 9-item instrument that assesses the presence and severity of depression by asking respondents how much they were bothered by depression symptoms during the past two weeks. ${ }^{35}$ The items' response options and their corresponding scores were: nearly every day (3), more than half of the days (2), several days (1), or not at all (0), yielding a total score ranging from 0 to 27. The severity of depression and its corresponding scores on the PHQ-9 were minimal depression (0-4), mild depression (5-9), moderate depression (10-14), moderately severe depression (15-19), and severe depression (20-27). ${ }^{34}$ The Arabic version of the GAD-7 questionnaire has 7 items that assess the presence and severity of anxiety by also asking respondents how much they were bothered by anxiety symptoms during the past two weeks. ${ }^{35}$ The response options and their corresponding scores were: nearly every day (3), more than half of the days (2), several days (1), or not at all (0), yielding a total score ranging from 0 to 21 . The levels of anxiety and their corresponding scores were minimal anxiety (0-4), mild anxiety (5-9), moderate anxiety (10-14), and severe anxiety (15-21). ${ }^{36}$ The Arabic version of the EMIC stigma scale is an easy-to-administer validated tool to assess an individual's perception of stigma by people nearby or close to them because of the individual's specific health condition. ${ }^{33}$ Originally, the EMIC stigma scale was developed by Weiss et al as a tool to understand the stigma related to leprosy. Afterwards, its validity and reliability as a stigma assessment tool have been tested and confirmed in different settings and for other infectious and chronic diseases. ${ }^{38-40}$ The EMIC stigma scale consists of 15 items related to the participant's feelings of stigma and are scored on a 4-point scale: Yes (3), Possibly (2), Uncertain (1), and No (0), with reverse scoring of item 2. The EMIC total score ranges from 0 to 36 , with a higher score indicating a higher level of stigma. ${ }^{37}$

\section{Data Collection}

In addition to the clinical data generated by participants' responses to the items in the instruments, we collected data on sociodemographic characteristics (eg, age, gender, marital status, number of family members living with the recovered patient, region of residence, employment status, monthly income, and educational level), comorbidities and family history of mental illness. Moreover, participants' health literacy was measured using the Arabic version of the Singleitem Literacy Screener (SILS). ${ }^{41}$ Participants were asked about their perceived need for help in order to understand a prescription medication leaflet; they were instructed to respond to the question with one of five possible answers (never, rarely, sometimes, often, or always). The answers "always, often, or sometimes" indicated limited or marginal health literacy, and "rarely or never" suggested adequate health literacy. ${ }^{42}$ 


\section{Statistical Analysis}

Descriptive statistics, including means with standard deviations and frequencies with percentages were used to report participants' characteristics and total scores on the PHQ-9, GAD-7, and EMIC scales. Moreover, two multiple linear regression models were conducted to explore whether participants' stigma experience (EMIC score) was associated with their level of depression (PHQ-9 score) or anxiety (GAD-7 score). Other socioeconomic and medical factors that may affect mental health, such as age, gender, health literacy, a chronic health condition, and education were included in the multiple regression models. Based on a medium effect size (Cohen's $\left.\mathrm{f}^{2}=0.15\right), \alpha=0.05, \beta=0.2$, power of $80 \%$, and nine independent variables for a multiple linear regression, the minimum sample size was estimated to be 104 participants. The significance level was set at $\alpha<0.05$. All statistical analyses were performed using $\mathrm{SAS}^{\circledR}$ statistical software version $9.4\left(\mathrm{SAS}^{\circledR}\right.$ institute, Cary, NC, USA).

\section{Results}

\section{Participants' Baseline Characteristics}

Of the 211 adult patients who met the inclusion criteria and were contacted by the team, $174(82.46 \%)$ consented to participate in the study and were interviewed. The mean age of the participants was $31.58( \pm 10.2)$ years and $54.6 \%$ of them were female. Most of the participants were Saudi citizens, had an adequate level of health literacy, and only $17.2 \%$ had a chronic health condition. In addition, more than half of the participants were married, living in the central region, and had at least a bachelor's degree. The participants' characteristics are summarized in Table 1.

\section{Depression, Anxiety, and Stigma Levels}

The participants' mean PHQ-9 (depression) score was 7.53 ( \pm 5.04$)$ and their mean GAD-7 (anxiety) score was 3.77 ( \pm 4.47$)$. More than two-thirds of the participants $(68.39 \%)$ had at least mild depression, whereas only $29.89 \%$ of them had scores indicating at least mild anxiety. Moreover, about $12 \%$ of participants suffered from moderately severe to severe depression when they were infected with COVID-19, and 13\% reported having anxiety during their COVID-19 infection. The participants' mean EMIC score for stigma was 5.91 ( \pm 5.21$)$, implying a low level of stigma (Table 2).

\section{Association Between Participants' Scores on the Measures of Depression (PHQ-9) and Stigma (EMIC)}

Participants with higher EMIC scores were more likely to have higher PHQ-9 scores $(\beta=0.30,95 \%$ CI $=0.16-0.44$, $p<0.0001$ ), controlling for age, gender, education, health literacy, and the presence of a chronic health condition. In addition, female participants were more likely to have higher PHQ-9 scores than their male counterparts ( $\beta=3.071,95 \%$ $\mathrm{CI}=1.62-4.51 ; p<0.0001)$. The association between the PHQ-9 and EMIC scores is presented in Table 3.

\section{Association Between Participants' Scores on the Measures of Anxiety (GAD-7) and Stigma (EMIC)}

Participants with higher EMIC scores were more likely to have higher GAD-7 scores $(\beta=0.26,95 \% \mathrm{CI}=0.13-0.38$, $p<0.0001$ ), controlling for age, gender, education, health literacy, and the presence of a chronic health condition. Similarly, female participants were more likely to have higher GAD-7 scores than their male counterparts $(\beta=1.86$, $95 \% \mathrm{CI}=0.58-3.15 ; p<0.004)$. The presence of a chronic health condition was positively associated with GAD-7 scores $(\beta=1.73,95 \% \mathrm{CI}=0.04-3.42, p=0.043)$. The association between the GAD-7 and EMIC scores is presented in Table 4 .

\section{Discussion}

This study aimed to explore the potential associations of depression and anxiety with feelings of stigma among individuals who have recovered from COVID-19 infection in a conservative culture, such as Saudi Arabia. In this study, the prevalence of depression among the participants was significantly higher than anxiety, as more than two-thirds of them were found to have mild to severe depression, and only $30 \%$ had mild to severe anxiety during their COVID-19 
Table I Participants' Characteristics

\begin{tabular}{|c|c|}
\hline Characteristic & Mean ( \pm SD) $/ \mathrm{n}(\%)^{\mathrm{a}}$ \\
\hline \multicolumn{2}{|l|}{ Sex } \\
\hline Male & $79(45.40)$ \\
\hline Female & $95(54.60)$ \\
\hline Age, Mean ( \pm SD) & $31.58( \pm 10.27)$ \\
\hline \multicolumn{2}{|l|}{ Annual income } \\
\hline$<\$ 9600$ & $73(42.7)$ \\
\hline$\$ 9600-\$ 19,200$ & $23(13.5)$ \\
\hline$>\$ 19,200-\$ 31,992$ & II (6.4) \\
\hline$>\$ 31,992-\$ 48,000$ & $24(14.0)$ \\
\hline$>\$ 48,000-\$ 63,996$ & $21(12.3)$ \\
\hline$>\$ 63,996-\$ 79,992$ & $14(8.2)$ \\
\hline Refrained from answering & $4(2.3)$ \\
\hline \multicolumn{2}{|l|}{ Education } \\
\hline No formal education & $3(1.7)$ \\
\hline Elementary school & $4(2.3)$ \\
\hline Intermediate school & II (6.3) \\
\hline Secondary school & $36(20.8)$ \\
\hline Associate degree & $22(12.6)$ \\
\hline Bachelor's degree & $94(54.0)$ \\
\hline Postgraduate degree (eg, master's or doctorate degree) & $4(2.3)$ \\
\hline Participants with limited health literacy & $27(15.5)$ \\
\hline \multicolumn{2}{|l|}{ Marital status } \\
\hline Single & $75(43.1)$ \\
\hline Married & $92(52.9)$ \\
\hline Separated/Divorced & $3(1.7)$ \\
\hline Widow & $4(2.3)$ \\
\hline \multicolumn{2}{|l|}{ Nationality } \\
\hline Saudi & $152(87.4)$ \\
\hline Non-Saudi & $22(12.6)$ \\
\hline \multicolumn{2}{|l|}{ Region of residence } \\
\hline Central & $92(52.9)$ \\
\hline Western & $23(13.2)$ \\
\hline Eastern & $4 I(23.6)$ \\
\hline Southern & $16(9.2)$ \\
\hline Northern & $2(1.1)$ \\
\hline \multicolumn{2}{|l|}{ Chronic health condition } \\
\hline Diabetes & $9(5.2)$ \\
\hline Dyslipidemia & $4(2.3)$ \\
\hline Cardiovascular disease & $7(4.0)$ \\
\hline Asthma & $7(4.0)$ \\
\hline Hypothyroidism & $3(1.7)$ \\
\hline
\end{tabular}

Note: ${ }^{\mathrm{a} D a t a}$ are presented as mean $( \pm \mathrm{SD})$ or frequency $(\%)$.

Abbreviations: $n$, number; SD, standard deviation.

infection. However, higher stigma scores were positively associated with higher levels of depression and anxiety, even after adjusting for multiple covariates.

During the pandemic, several preventive measures were implemented to minimize infection transmission, such as social distancing, isolation, and lockdowns. These measures, combined with the continuous spread of the virus and growing death rates could have negatively affected the community's mental health. Therefore, the relatively high prevalence rates of depression and anxiety among the participants, all of whom had been infected with COVID-19, were expected during the infection period, due to the disease process. The preventive measures that were taken during 
Table 2 Participants' Mean Scores on the EMIC, PHQ-9, and GAD-7 and the Distributions of Levels of Depression (PHQ-9 Scores) and General Anxiety (GAD-7 Scores)

\begin{tabular}{|c|c|c|}
\hline Level & Score Range & a Mean (士SD)/n (\%) \\
\hline EMIC, Mean ( $\pm S D)$ & $(0-36)$ & $5.91( \pm 5.21)$ \\
\hline PHQ-9, Mean (士SD) & $(0-27)$ & $7.53( \pm 5.04)$ \\
\hline Minimal depression & $1-4$ & $55(31.6 I)$ \\
\hline Mild depression & $5-9$ & $62(35.63)$ \\
\hline Moderate depression & $10-14$ & $36(20.69)$ \\
\hline Moderately severe depression & $15-19$ & $19(10.92)$ \\
\hline Severe depression & $20-27$ & $2(1.15)$ \\
\hline GAD-7, Mean (士SD) & $(0-2 I)$ & $3.77( \pm 4.47)$ \\
\hline Minimal anxiety & $0-4$ & $122(70.11)$ \\
\hline Mild anxiety & $5-9$ & $29(16.67)$ \\
\hline Moderate anxiety & $10-14$ & $16(9.20)$ \\
\hline Severe anxiety & $|5-2|$ & $7(4.02)$ \\
\hline
\end{tabular}

Note: ${ }^{a}$ Data are presented as mean $( \pm S D)$ or frequency $(\%)$.

Abbreviations: EMIC, Explanatory Model Interview Catalogue stigma scale; PHQ-9, Patient Health Questionnaire-9; and GAD-7, Generalized Anxiety Disorder-7 questionnaire; SD, standard deviation.

Table 3 Results of the Multiple Linear Regression for the Association Between the PHQ-9 and EMIC Scores

\begin{tabular}{|c|c|c|c|c|}
\hline Variable & $\beta$ Estimate & \multicolumn{2}{|l|}{$95 \% \mathrm{Cl}$} & $P$-value \\
\hline EMIC & 0.303 & 0.166 & 0.441 & $<0.000 I^{*}$ \\
\hline Age & 0.032 & -0.041 & 0.105 & 0.392 \\
\hline Gender (Female) & 3.071 & 1.629 & 4.514 & $<0.000 I^{*}$ \\
\hline Education & 0.319 & -0.260 & 0.899 & 0.278 \\
\hline Health literacy & -0.188 & -2.152 & 1.776 & 0.850 \\
\hline Presence of a chronic health condition (eg, diabetes) & -0.30227 & -2.193 & 1.588 & 0.753 \\
\hline
\end{tabular}

Note: $* p<0.05$.

Abbreviations: 95\% Cl, 95\% confidence interval; PHQ-9, Patient Health Questionnaire-9; EMIC, Explanatory Model Interview Catalogue stigma scale.

Table 4 Results of the Multiple Linear Regression for the Association Between the GAD-7 and EMIC Scores

\begin{tabular}{|c|c|c|c|c|}
\hline Variable & $\beta$ Estimate & \multicolumn{2}{|l|}{$95 \% \mathrm{Cl}$} & $p$-value \\
\hline EMIC & 0.261 & 0.138 & 0.384 & $<0.000 I^{*}$ \\
\hline Age & -0.020 & -0.085 & 0.045 & 0.542 \\
\hline Gender (Female) & 1.869 & 0.582 & 3.156 & $0.004 *$ \\
\hline Education & 0.314 & -0.204 & 0.831 & 0.232 \\
\hline Health literacy & -0.664 & -2.416 & 1.089 & 0.456 \\
\hline Presence of a chronic health condition (eg, diabetes) & 1.734 & 0.048 & 3.421 & $0.043 *$ \\
\hline
\end{tabular}

Note: $* p$-value $<0.05$

the pandemic had some significant effects on the physical and psychological health and social life of humans worldwide. A nationwide study of the general population of Saudi Arabia revealed similar levels of depression $(70.3 \%)$, but a much higher rate of anxiety symptoms (mild $=73.5 \%$, moderate $=19.3 \%$, and severe $=7.3 \%) .{ }^{43}$ However, that study was conducted between March and April 2020, which was the early phase of the COVID-19 outbreak in Saudi Arabia. As result, a higher prevalence of anxiety was predicted because that period was associated with a nationwide lockdown and poor understanding of the virus. However, Joseph et al found a comparable level of moderate to severe anxiety symptoms among the general population (17.8\% vs $13.2 \%$ in our study) ${ }^{44}$ Importantly, the result of the current study showed that higher stigma scores were positively correlated with depression and 
anxiety levels. These possible associations of depression and anxiety with stigma were also reported in the literature in different settings and patient populations. ${ }^{23,45}$ However, no studies have examined the associations of depression and anxiety with stigma among patients with COVID-19. In addition, a significant association was found between being female and having increased mean scores for depression $(\beta=3.071)$ and anxiety $\beta=1.86)$, which is similar to the findings reported in local and international studies. ${ }^{46-48}$ Females are generally more likely than their male counterparts to report symptoms of depression and anxiety which is hypothetically related to hormonal fluctuations, higher sensitivity to stress, and some socioeconomic factors, such as higher income in favor of their male counterparts. ${ }^{49}$

Compared to other previously published studies, the participants in this study were less likely to experience depression and anxiety symptoms. For example, a study that evaluated the general population's mental health in Brazil during the COVID-19 outbreak indicated that $67.7 \%$ of participants had moderate to severe depression and $84.7 \%$ had anxiety symptoms. ${ }^{50}$ Another study that assessed depression and anxiety among the general population of China found that $4.3 \%$ of participants suffered from severe depression while $8.4 \%$ experienced severe anxiety. ${ }^{48}$ Furthermore, the prevalence of severe anxiety among the general Iranian population during the COVID-19 outbreak was $19.1 \% .^{51}$ Nevertheless, a study conducted in Vietnam reported much lower rates of anxiety (4.9\%) and depression (7.0\%), compared to our estimates. ${ }^{52}$ Many factors may explain the wide range of reported prevalence rates of depression and anxiety in different countries. This can be due to variation in the measures used to assess depression and anxiety in these studies, which were conducted during the COVID-19 outbreak. Furthermore, different approaches to categorizing levels of depression and anxiety were used in the different studies, which might have increased the variability of the results between the studies. Moreover, the timing of the study and the stage of the pandemic are other critical factors. It is expected that the psychological symptoms would be more prevalent during the early stages of the pandemic, as little information was known about the virus and the disease. For instance, in a study that evaluated depression and anxiety symptoms among 58 hospitalized COVID-19 patients in a universityaffiliated hospital in New York city, the prevalence of depression and anxiety upon admission were $29 \%$ and $36 \%$, respectively. However, the prevalence of depression and anxiety symptoms dropped to $20 \%$ and $9 \%$, respectively, after two weeks of follow-up. ${ }^{53}$ Therefore, it would be interesting to know the impact of time on the symptoms of depression, anxiety, and stigma among the participants in this study couple of months or a year later.

The findings of the present study highlight the adverse psychological effects of the COVID-19 pandemic among patients who recovered from COVID-19. Our results emphasize the need to adopt strategies that can address and alleviate these adverse consequences. For instance, psychiatric screening should be implemented regularly to detect depression and anxiety in the early stages, which could lead to the use of interventions that are more effective. Moreover, a global collaborative effort is required to develop a preparedness plan that can promote, protect, and address mental health during the current and future health crises.

This study has limitations that should be considered when interpreting our results. The cross-sectional design of this study precludes the ability to examine causal relationships between the study's variables. Furthermore, the present study did not evaluate participants' previous history of mental health disorders that may have existed prior to the pandemic. Although self-reported symptoms of anxiety and depression during the interview might not accurately reflect participants' actual mental health status, the study used validated assessment tools, which are commonly used in practice. In addition, this study's findings only provide a snapshot of the negative psychological effects of COVID-19 on individuals who recovered from it. Thus, the duration of the observed psychological effects after recovery was not assessed and should be examined in future studies. Lastly, the study was conducted several weeks after the patients' recovery from COVID-19, which increases the risk of recall bias.

\section{Conclusion}

Among the study's participants who had recently recovered from COVID-19 in Saudi Arabia, depression affected about two-thirds of them and anxiety affected one-third. Notably, stigma scores were significantly associated with increased levels of depression and anxiety, and females were more likely to be affected psychologically by COVID-19 infection. These findings indicate the need to implement strategies that will ensure the availability of psychosocial support to promote the mental well-being of patients during the current and future pandemics. Future studies with larger samples of 
COVID-19 patients, more diverse clinical presentations (eg, ICU admitted, hospitalized in non-intensive care units, moderate to mild symptoms, and asymptomatic), and prospective follow-up to examine the impact of time on symptoms of depression, anxiety, and stigma should be conducted.

\section{Data Sharing Statement}

The data will be available from the corresponding author upon reasonable request.

\section{Ethical Approval}

The Kingdom of Saudi Arabia's MOH approved the study's protocol (IRB No: 20-11E/17-06-2020), and the ethics committee waived the requirement for patients' written informed consent to participate in the study.

\section{Acknowledgments}

The authors acknowledge the financial support received from the Researchers Supporting Project number [RSP-2021/16], King Saud University, Riyadh, Saudi Arabia. The authors gratefully acknowledge Lamia Al-Zamel, Shatha Al-Thunayan, and Afnan Al-Rasheed for their contributions to the data collection process.

\section{Disclosure}

The authors declare that they have no financial or nonfinancial conflicts of interest in this work.

\section{References}

1. Ueda M, Nordström R, Matsubayashi T. Suicide and mental health during the COVID-19 pandemic in Japan. J Public Health. 2021 ;fdab113. doi:10.1093/pubmed/fdab113

2. Nicola M, Alsafi Z, Sohrabi C, et al. The socio-economic implications of the coronavirus pandemic (COVID-19): a review. Int J Surg. 2020;78:185-193.

3. Hotopf M, Bullmore E, O'Connor RC, Holmes EA. The scope of mental health research during the COVID-19 pandemic and its aftermath. $\mathrm{Br}$ J Psychiatry. 2020;217(4):540-542.

4. Srivastava N, Saxena SK. Prevention and Control Strategies for SARS-CoV-2 Infection. In: Saxena SK, editor. Coronavirus Disease 2019 (COVID19): Epidemiology, Pathogenesis, Diagnosis, and Therapeutics. Singapore: Springer; 2020:127-140.

5. AlRasheed MM, Alkadir AM, Bin Shuqiran KI, Al-Aqeel S, Jahrami HA, BaHammam AS. The Impact of Quarantine on Sleep Quality and Psychological Distress During the COVID-19 Pandemic. Nat Sci Sleep. 2021;13:1037-1048.

6. Bueno-Notivol J, Gracia-García P, Olaya B, Lasheras I, López-Antón R, Santabárbara J. Prevalence of depression during the COVID-19 outbreak: a meta-analysis of community-based studies. Int J Clin Health Psychol. 2021;21(1):100196.

7. Javed B, Sarwer A, Soto EB, Mashwani Z-U-R. The coronavirus (COVID-19) pandemic's impact on mental health. Int J Health Plann Manage. 2020;35(5):993-996.

8. Hall RC, Hall RC, Chapman MJ. The 1995 Kikwit Ebola outbreak: lessons hospitals and physicians can apply to future viral epidemics. Gen Hosp Psychiatry. 2008;30(5):446-452.

9. Xiang YT, Yang Y, Li W, et al. Timely mental health care for the 2019 novel coronavirus outbreak is urgently needed. Lancet Psychiatry. 2020;7 (3):228-229.

10. Zhang J, Lu H, Zeng H, et al. The differential psychological distress of populations affected by the COVID-19 pandemic. Brain Behav Immun. 2020;87:49-50. doi:10.1016/j.bbi.2020.04.031

11. Spijker J, Graaf R, Bijl R, Beekman A, Ormel J, Nolen W. Functional disability and depression in the general population. Results from the Netherlands Mental Health Survey and incidence Study (NEMENESIS). Acta Psychiatr Scand. 2004;110:208-214.

12. Ustün TB, Ayuso-Mateos JL, Chatterji S, Mathers C, Murray CJ. Global burden of depressive disorders in the year $2000 . B r J$ Psychiatry. 2004;184:386-392.

13. Murray CJL, Lopez AD. Evidence-Based Health Policy-Lessons from the Global Burden of Disease Study. Science. 1996;274(5288):740.

14. Li Z, Ruan M, Chen J, Fang Y. Major Depressive Disorder: advances in Neuroscience Research and Translational Applications. Neurosci Bull. 2021;37(6):863-880.

15. DeMartini J, Patel G, Fancher TL. Generalized Anxiety Disorder. Ann Intern Med. 2019;170(7):49-64.

16. Muhammad Gadit AA, Mugford G. Prevalence of Depression among Households in Three Capital Cities of Pakistan: need to Revise the Mental Health Policy. PLoS One. 2007;2(2):e209.

17. Xiong J, Lipsitz O, Nasri F, et al. Impact of COVID-19 pandemic on mental health in the general population: a systematic review. $J$ Affect Disord. 2020;277:55-64.

18. Huang Y, Zhao N. Generalized anxiety disorder, depressive symptoms and sleep quality during COVID-19 outbreak in China: a web-based cross-sectional survey. Psychiatry Res. 2020;288:112954.

19. Shevlin M, McBride O, Murphy J, et al. Anxiety, depression, traumatic stress and COVID-19-related anxiety in the UK general population during the COVID-19 pandemic. BJPsych Open. 2020;6(6):e125.

20. Bagcchi S. Stigma during the COVID-19 pandemic. Lancet Infect Dis. 2020;20(7):782. 
21. Ociskova M, Prasko J, Kamaradova D, Grambal A, Sigmundova Z. Individual correlates of self-stigma in patients with anxiety disorders with and without comorbidities. Neuropsychiatr Dis Treat. 2015;11:1767-1779.

22. Pyne J, Kuc E, Schroeder P, Fortney J, Edlund M, Sullivan G. Relationship Between Perceived Stigma and Depression Severity. J Nerv Ment Dis. 2004;192:278-283.

23. Charles B, Jeyaseelan L, Pandian AK, Sam AE, Thenmozhi M, Jayaseelan V. Association between stigma, depression and quality of life of people living with HIV/AIDS (PLHA) in South India - a community based cross sectional study. BMC Public Health. 2012;12(1):463.

24. Goffman E. Stigma: notes on the management of spoiled identity Garden City. NY Anchor Books. 1963.

25. Schnyder N, Panczak R, Groth N, Schultze-Lutter F. Association between mental health-related stigma and active help-seeking: systematic review and meta-analysis. Br J Psychiatry. 2017;210(4):261-268.

26. Rogers JP, Chesney E, Oliver D, et al. Psychiatric and neuropsychiatric presentations associated with severe coronavirus infections: a systematic review and meta-analysis with comparison to the COVID-19 pandemic. Lancet Psychiatry. 2020;7(7):611-627.

27. Bo HX, Li W, Yang Y, et al. Posttraumatic stress symptoms and attitude toward crisis mental health services among clinically stable patients with COVID-19 in China. Psychol Med. 2021;51(6):1052-1053.

28. Wu T, Jia X, Shi H, et al. Prevalence of mental health problems during the COVID-19 pandemic: a systematic review and meta-analysis. $J$ Affect Disord. 2021;281:91-98.

29. Guo Q, Zheng Y, Shi J, et al. Immediate psychological distress in quarantined patients with COVID-19 and its association with peripheral inflammation: a mixed-method study. Brain Behav Immun. 2020;88:17-27.

30. Holmes EA, O'Connor RC, Perry VH, et al. Multidisciplinary research priorities for the COVID-19 pandemic: a call for action for mental health science. Lancet Psychiatry. 2020;7(6):547-560.

31. Hossain MM, Sultana A, Purohit N. Mental health outcomes of quarantine and isolation for infection prevention: a systematic umbrella review of the global evidence. Epidemiol Health. 2020;42:e2020038.

32. Hao F, Tan W, Jiang L, et al. Do psychiatric patients experience more psychiatric symptoms during COVID-19 pandemic and lockdown? A case-control study with service and research implications for immunopsychiatry. Brain Behav Immun. 2020;87:100-106.

33. Al-Zamel LA, Al-Thunayan SF, Al-Rasheed AA, et al. Validation and Cultural Adaptation of Explanatory Model Interview Catalogue (EMIC) in Assessing Stigma among Recovered Patients with COVID-19 in Saudi Arabia. Int J Environ Res Public Health. 2021;18(16):8261.

34. Kroenke K, Spitzer RL, Williams JB, The PHQ-9. validity of a brief depression severity measure. J Gen Intern Med. 2001;16(9):606-613.

35. AlHadi AN, AlAteeq DA, Al-Sharif E, et al. An arabic translation, reliability, and validation of Patient Health Questionnaire in a Saudi sample. Ann Gen Psychiatry. 2017;16:32.

36. Spitzer RL, Kroenke K, Williams JB, Löwe B. A brief measure for assessing generalized anxiety disorder: the GAD-7. Arch Intern Med. 2006;166 (10):1092-1097.

37. Weiss M. Explanatory Model Interview Catalogue (EMIC): framework for Comparative Study of Illness. Transcult Psychiatry. 1997;34 (2):235-263.

38. Raguram R, Raghu TM, Vounatsou P, Weiss MG. Schizophrenia and the cultural epidemiology of stigma in Bangalore, India. J Nerv Ment Dis. 2004;192(11):734-744.

39. Mukherjee S, Mukhopadhyay DK. Stigma towards mental illness: a hospital-based cross-sectional study among caregivers in West Bengal. Indian J Public Health. 2018;62(1):15-20.

40. Oladele DA, Balogun MR, Odeyemi K, Salako BL. A Comparative Study of Knowledge, Attitude, and Determinants of Tuberculosis-Associated Stigma in Rural and Urban Communities of Lagos State, Nigeria. Tuberc Res Treat. 2020;2020:1964759.

41. Al-Jumaili AA, Al-Rekabi MD, Sorofman B. Evaluation of instruments to assess health literacy in Arabic language among Iraqis. Res Social Adm Pharm. 2015;11(6):803-813.

42. Morris NS, MacLean CD, Chew LD, Littenberg B. The Single Item Literacy Screener: evaluation of a brief instrument to identify limited reading ability. BMC Fam Pract. 2006;7(1):21.

43. Alyami HS, Naser AY, Dahmash EZ, Alyami MH, Alyami MS. Depression and anxiety during the COVID-19 pandemic in Saudi Arabia: a cross-sectional study. Int J Clin Pract. 2021;75(7):e14244-e14244.

44. Joseph R, Lucca JM, Alshayban D, Alshehry YA. The immediate psychological response of the general population in Saudi Arabia during COVID-19 pandemic: a cross-sectional study. J Infect Public Health. 2021;14(2):276-283.

45. Pellet J, Golay P, Nguyen A, et al. The relationship between self-stigma and depression among people with schizophrenia-spectrum disorders: a longitudinal study. Psychiatry Res. 2019;275:115-119.

46. Alamri HS, Algarni A, Shehata SF, et al. Prevalence of Depression, Anxiety, and Stress among the General Population in Saudi Arabia during Covid-19 Pandemic. Int J Environ Res Public Health. 2020;17(24):9183.

47. Qiu J, Shen B, Zhao M, Wang Z, Xie B, Xu Y. A nationwide survey of psychological distress among Chinese people in the COVID-19 epidemic: implications and policy recommendations. Gen Psychiatr. 2020;33(2):e100213.

48. Wang C, Pan R, Wan X, et al. Immediate Psychological Responses and Associated Factors during the Initial Stage of the 2019 Coronavirus Disease (COVID-19) Epidemic among the General Population in China. Int J Environ Res Public Health. 2020;17(5):1729.

49. Albert PR. Why is depression more prevalent in women? J Psychiatry Neurosci. 2015;40(4):219-221.

50. Goularte JF, Serafim SD, Colombo R, Hogg B, Caldieraro MA, Rosa AR. COVID-19 and mental health in Brazil: psychiatric symptoms in the general population. J Psychiatr Res. 2021;132:32-37.

51. Moghanibashi-Mansourieh A. Assessing the anxiety level of Iranian general population during COVID-19 outbreak. Asian J Psychiatr. 2020;51:102076.

52. Le HT, Lai AJX, Sun J, et al. Anxiety and Depression Among People Under the Nationwide Partial Lockdown in Vietnam. Frontiers in Public Health. 2020;8(656):589359.

53. Parker C, Shalev D, Hsu I, et al. Depression, Anxiety, and Acute Stress Disorder Among Patients Hospitalized With COVID-19: a Prospective Cohort Study. J Acad Consult Liaison Psychiatry. 2021;62(2):211-219. 


\section{Publish your work in this journal}

Psychology Research and Behavior Management is an international, peer-reviewed, open access journal focusing on the science of psychology and its application in behavior management to develop improved outcomes in the clinical, educational, sports and business arenas. Specific topics covered in the journal include: Neuroscience, memory and decision making; Behavior modification and management; Clinical applications; Business and sports performance management; Social and developmental studies; Animal studies. The manuscript management system is completely online and includes a very quick and fair peer-review system, which is all easy to use. Visit http://www.dovepress.com/testimonials.php to read real quotes from published authors.

Submit your manuscript here: https://www.dovepress.com/psychology-research-and-behavior-management-journal 